\title{
Approaching the Hartree-Fock limit by perturbative methods
}

\author{
Jia Deng, Andrew T. B. Gilbert, and Peter M. W. Gill ${ }^{\mathrm{a})}$ \\ Research School of Chemistry, The Australian National University, \\ Australian Capital Territory 0200, Australia
}

(Received 1 May 2009; accepted 20 May 2009; published online 15 June 2009)

\begin{abstract}
We describe perturbative methods for improving finite-basis Hartree-Fock calculations toward the complete-basis limit. The best method appears to offer quadratic error reduction and preliminary numerical applications demonstrate that remarkably accurate Hartree-Fock energies can be obtained. (C) 2009 American Institute of Physics. [DOI: 10.1063/1.3152864]
\end{abstract}

\section{INTRODUCTION}

Nonrelativistic $a b$ initio quantum chemistry is a twodimensional convergence problem, in which the first dimension corresponds to the correlation treatment and the second to the basis set. The solution of the Schrödinger equation ${ }^{1}$ is found by converging both limits simultaneously, which is known as full configuration interaction at the complete basis set (CBS) limit.

Our interest here, however, is Hartree-Fock (HF) with a $\mathrm{CBS}$, a theoretical model that is sometimes called the HF limit. Unfortunately, because a CBS is necessarily infinite, such calculations are impossible in practice and even nearCBS calculations are limited to small systems because of the high scaling of the computational cost with basis set size.

Basis set extrapolation offers one route to estimating the $\mathrm{HF} / \mathrm{CBS}$ energy and is now widely used..$^{2-10}$ However, it has a number of weaknesses: it lacks a rigorous theoretical foundation, its error cannot be rigorously bounded, extrapolated energies are nonvariational, and its reliability depends on the use of large basis sets.

We favor a different pathway. It is well known that the energy from a small-basis HF calculation is often close to the CBS limit and we therefore believe that a perturbative approach is the natural framework for estimating the small difference between them.

In this communication, we study three approaches (HF perturbative corrections or HFPCs) that estimate the HF/CBS energy by diagonalizing the Fock potential from a small (primary) basis in a near-complete (secondary) basis. These are related to the various dual-basis schemes that have been developed by King and co-workers, ${ }^{11,12}$ Jurgens-Lutovsky and Almlöf, ${ }^{13}$ Wolinski and Pulay, ${ }^{14}$ and Head-Gordon and co-workers, ${ }^{15-18}$ but our methods retain all quadratic contributions to the energy correction. Moreover, our best methods remove a previously overlooked self-interaction error and achieve a quadratic reduction in the energy error. Atomic units are used throughout.

\section{HARTREE-FOCK THEORY}

The exact RHF orbitals $\psi_{i}$ of a $2 m$-electron system satisfy the integrodifferential eigenequation,

\footnotetext{
${ }^{a)}$ Electronic mail: peter.gill@anu.edu.au.
}

$$
\mathbf{F} \psi_{i}=\varepsilon_{i} \psi_{i}, \quad i=1,2, \ldots, m,
$$

where, using standard notation, ${ }^{19}$ the Fock operator is

$$
\mathbf{F}=\mathbf{h}+\sum_{j}^{m}\left(2 \mathbf{J}_{j}-\mathbf{K}_{j}\right),
$$

the one-electron operator $\mathbf{h}$ is independent of the orbitals, and the Coulomb and exchange operators are defined by

$$
\begin{aligned}
& \mathbf{J}_{j} f(\boldsymbol{r})=f(\boldsymbol{r}) \int \frac{\psi_{j}\left(\boldsymbol{r}^{\prime}\right) \psi_{j}\left(\boldsymbol{r}^{\prime}\right)}{\left|\boldsymbol{r}-\boldsymbol{r}^{\prime}\right|} d \boldsymbol{r}^{\prime}, \\
& \mathbf{K}_{j} f(\boldsymbol{r})=\psi_{j}(\boldsymbol{r}) \int \frac{\psi_{j}\left(\boldsymbol{r}^{\prime}\right) f\left(\boldsymbol{r}^{\prime}\right)}{\left|\boldsymbol{r}-\boldsymbol{r}^{\prime}\right|} d \boldsymbol{r}^{\prime} .
\end{aligned}
$$

The exact RHF energy from these orbitals is

$$
E=2 \sum_{i}^{m} h_{i i}+\sum_{i j}^{m}\left(2 J_{i j}-K_{i j}\right) .
$$

It is easy to see from Eqs. (3) and (4) that

$$
\left(\mathbf{J}_{i}-\mathbf{K}_{i}\right) \psi_{i}=0
$$

and, because of this, HF theory avoids the self-interaction error that plagues many density functional models.

In practice, however, most $\mathrm{HF}$ calculations are performed in an incomplete (primary) basis of $n$ functions using variational formalisms introduced by Roothaan, ${ }^{20} \mathrm{Hall}^{21}$ and Pople and Nesbet. ${ }^{22}$ These yield approximate orbitals $\psi_{i}^{[0]}$ and an associated approximate energy,

$$
E^{[0]}=2 \sum_{i}^{m} h_{i i}^{[0]}+\sum_{i j}^{m}\left(2 J_{i j}^{[0]}-K_{i j}^{[0]}\right),
$$

but, contrary to popular misconception, the $\psi_{i}^{[0]}$ are not eigenfunctions of their Fock operator,

$$
\mathbf{F}^{[0]}=\mathbf{h}+\sum_{j}^{m}\left(2 \mathbf{J}_{j}^{[0]}-\mathbf{K}_{j}^{[0]}\right),
$$

unless the basis exactly spans the $\psi_{i}$. Our HFPC approach is motivated by the suspicion that the true eigenfunctions $\psi_{i}^{[1]}$ of $\mathbf{F}^{[0]}$, which satisfy 
TABLE I. Formal costs of steps in the three HFPC schemes ( $n$ is the size of the primary basis and $N$ is the size of the secondary basis).

\begin{tabular}{lccc}
\hline \hline & HFPC[1] & HFPC[2] & HFPC[3] \\
\hline Primary SCF & $O\left(n^{4}\right)$ & $O\left(n^{4}\right)$ & $O\left(n^{4}\right)$ \\
Form $S_{a b}$ & $O\left(N^{2}\right)$ & $O\left(N^{2}\right)$ & $O\left(N^{2}\right)$ \\
Form $X_{a b}$ & $O\left(N^{3}\right)$ & $O\left(N^{3}\right)$ & $O\left(N^{3}\right)$ \\
Form $F_{i}$ & $O\left(n^{2} N^{2}\right)$ & $m O\left(n^{2} N^{2}\right)$ & $m O\left(n^{2} N^{2}\right)$ \\
Form $F_{i}^{\prime}$ & $O\left(N^{3}\right)$ & $m O\left(N^{3}\right)$ & $m O\left(N^{3}\right)$ \\
Diagonalize $F_{i}^{\prime}$ & $O\left(N^{3}\right)$ & $m O\left(N^{3}\right)$ & $m O\left(N^{3}\right)$ \\
Form $C_{a i}$ & $m O\left(N^{2}\right)$ & $m O\left(N^{2}\right)$ & $m O\left(N^{2}\right)$ \\
Form $\left\langle\psi_{i}^{[2]} \mid \psi_{j}^{[2]}\right\rangle$ & & & $m^{2} O(N)$ \\
Form $X_{i j}$ & & & $m^{3} O(1)$ \\
Orthogonalize $C_{a i}$ & & $O\left(N^{4}\right)$ & $m^{2} O(N)$ \\
Form $E^{[1,2 \text { or 3] }}$ & $O\left(N^{4}\right)$ & & $O\left(N^{4}\right)$ \\
\hline \hline
\end{tabular}

$$
\mathbf{F}^{[0]} \psi_{i}^{[1]}=\varepsilon_{i}^{[1]} \psi_{i}^{[1]},
$$

are superior to the $\psi_{i}^{[0]}$ and should yield a better energy. To explore this, we diagonalize $\mathbf{F}^{[0]}$ in a near-complete (secondary) basis of $N$ functions and then examine three ways to estimate $E$ from the resulting $\psi_{i}^{[1]}$.

\section{PERTURBATIVE CORRECTIONS}

\section{A. HFPC $[1]$}

In our simplest and cheapest approach, HFPC[1], we find the energy $E^{[1]}$ directly from the orbitals obtained by diagonalizing $\mathbf{F}^{[0]}$ in the secondary basis. The key weakness of this is that, although the $\psi_{i}^{[1]}$ are eigenfunctions of $\mathbf{F}^{[0]}$, they are not self-consistent with it and so

$$
\left(\mathbf{J}_{i}^{[0]}-\mathbf{K}_{i}^{[0]}\right) \psi_{i}^{[1]} \neq 0 .
$$

Each of the $\psi_{i}^{[1]}$ is therefore corrupted by self-interaction and when they are used to compute the energy

$$
E^{[1]}=2 \sum_{i}^{m} h_{i i}^{[1]}+\sum_{i j}^{m}\left(2 J_{i j}^{[1]}-K_{i j}^{[1]}\right),
$$

it, too, necessarily inherits a self-interaction error.

Table I summarizes the required steps, using $\mu$ and $\nu$ to index primary basis functions, $a$ and $b$ to index secondary basis functions, and $i$ and $j$ to index molecular orbitals. As the table shows, we compute the overlap $S_{a b}$ and orthonormalization $X_{a b}$ matrices in the secondary basis, form Coulomb $(\mu \nu \mid a b)$ and exchange $(\mu a \mid \nu b)$ integrals ${ }^{23}$ to construct the Fock matrix $F$ of the primary potential in the secondary basis, transform $F$ into an orthogonal basis, diagonalize $F^{\prime}$, back transform to form the MO coefficients $C_{a i}$, and, finally, compute the energy.

\section{B. HFPC[2]}

HFPC [2] addresses the shortcomings in HFPC [1] by removing the offending self-interaction terms and forming a different Fock operator,

$$
\mathbf{F}_{i}^{[0]}=\mathbf{F}^{[0]}-\left(\mathbf{J}_{i}^{[0]}-\mathbf{K}_{i}^{[0]}\right),
$$

for each orbital and then solving

$$
\mathbf{F}_{i}^{[0]} \psi_{i}^{[2]}=\varepsilon_{i}^{[2]} \psi_{i}^{[2]} .
$$

As Table I shows, additional work is required to form and diagonalize the $m$ Fock matrices, but the remainder of the algorithm is unchanged.

This is essentially the Perdew-Zunger self-interaction correction $^{24}$ and its key weakness is that the orbitals $\psi_{i}^{[2]}$ are eigenfunctions of different Fock operators and therefore no longer necessarily orthogonal. As a result, the use of the standard HF energy formula,

$$
E^{[2]}=2 \sum_{i}^{m} h_{i i}^{[2]}+\sum_{i j}^{m}\left(2 J_{i j}^{[2]}-K_{i j}^{[2]}\right),
$$

is not strictly justified and $E^{[2]}$ is not variational.

\section{HFPC $[3]$}

In HFPC[3], we solve the nonorthogonality problem by constructing a set of orthonormal orbitals $\psi_{i}^{[3]}$ that span the same space as the occupied $\psi_{i}^{[2]}$. As Table I shows, this involves the construction and diagonalization of the overlap matrix between the $\psi_{i}^{[2]}$, but the additional cost is modest. The orthonormalization reintroduces a tiny self-interaction error and the final energy is

$$
E^{[3]}=2 \sum_{i}^{m} h_{i i}^{[3]}+\sum_{i j}^{m}\left(2 J_{i j}^{[3]}-K_{i j}^{[3]}\right) .
$$

Because this expression is invariant to orbital rotations, $E^{[3]}$ is independent of the orthonormalization procedure. Like $E^{[1]}$, it is also variational.

\section{NUMERICAL RESULTS}

\section{A. Computational details}

Primary HF calculations used Jensen's polarizationconsistent basis sets ${ }^{25-27}$ and the Q-CHEM 3.2 package. ${ }^{28} \mathrm{Cal}-$ culations on $\mathrm{Li}$ and $\mathrm{N}$ were spin unrestricted.

HFPC calculations used MATHEMATICA 6.0 (Ref. 29) and our results are summarized in Table II. The secondary basis consisted of 45 even-tempered Gaussians ${ }^{30}$ for $\mathrm{He}, \mathrm{Li}$, and $\mathrm{Be}$ (augmented with five diffuse functions for $\mathrm{He}$ ) and similarly large basis sets from Jensen ${ }^{31}$ for $\mathrm{H}, \mathrm{N}$, and Ne. Though none of these bases is complete, ${ }^{32}$ they are nearly so and yield all of the digits in the "HF/CBS" energies in Table II. They are all much larger than the pc- 4 basis. Of course, for routine HFPC calculations on larger systems, it will be important to choose secondary bases that exploit the full potential of HFPC while remaining computationally tractable. For comparison, we also used the $\mathrm{HF} / \mathrm{pc}-3$ and $\mathrm{HF} / \mathrm{pc}-4$ energies to generate the extrapolated ${ }^{7}$ energy

$$
E_{\mathrm{ext}}=\frac{E_{\mathrm{HF} / \mathrm{pc}-4}-e^{-2.5} E_{\mathrm{HF} / \mathrm{pc}-3}}{1-e^{-2.5}} .
$$

\section{B. Singlet He atom}

The pc- 0 basis for He consists of only two $s$ functions and the HF/pc-0 energy lies $28 \mathrm{~m} E_{h}$ above the HF limit. However, even this relatively crude starting point yields re- 
TABLE II. Errors (hartrees) in $E^{[0]}, E^{[1]}, E^{[2]}, E^{[3]}$, and $E_{\text {ext }}$ for $\mathrm{He}, \mathrm{H}_{2}, \mathrm{Li}, \mathrm{Be}, \mathrm{N}$, and $\mathrm{Ne}$

\begin{tabular}{|c|c|c|c|c|c|c|c|c|}
\hline & & & & & rimary ba & & & \\
\hline & $\mathrm{HF} / \mathrm{CBS}$ & & pc-0 & pc-1 & $\mathrm{pc}-2$ & pc-3 & pc-4 & $\Delta E_{\mathrm{ext}}$ \\
\hline $\operatorname{He}\left({ }^{1} S\right)$ & $-2.861679995612^{\mathrm{a}}$ & $\Delta E^{[0]}$ & $3 \times 10^{-2}$ & $8 \times 10^{-3}$ & $7 \times 10^{-4}$ & $3 \times 10^{-5}$ & $5 \times 10^{-6}$ & $+3 \times 10^{-6}$ \\
\hline & & $\Delta E^{[1]}$ & $2 \times 10^{-4}$ & $6 \times 10^{-5}$ & $3 \times 10^{-6}$ & $6 \times 10^{-8}$ & $1 \times 10^{-8}$ & \\
\hline & & $\Delta E^{[2]}$ & $3 \times 10^{-5}$ & $7 \times 10^{-6}$ & $1 \times 10^{-7}$ & $2 \times 10^{-11}$ & $2 \times 10^{-12}$ & \\
\hline & & $\Delta E^{[3]}$ & $3 \times 10^{-5}$ & $7 \times 10^{-6}$ & $1 \times 10^{-7}$ & $2 \times 10^{-11}$ & $2 \times 10^{-12}$ & \\
\hline $\mathrm{He}\left({ }^{3} S\right)$ & $-2.17425078^{b}$ & $\Delta E^{[0]}$ & $1 \times 10^{-0}$ & $7 \times 10^{-1}$ & $2 \times 10^{-1}$ & $7 \times 10^{-2}$ & $4 \times 10^{-2}$ & $+4 \times 10^{-2}$ \\
\hline & & $\Delta E^{[1]}$ & $8 \times 10^{-2}$ & $2 \times 10^{-2}$ & $3 \times 10^{-2}$ & $1 \times 10^{-2}$ & $8 \times 10^{-3}$ & \\
\hline & & $\Delta E^{[2]}$ & $2 \times 10^{-3}$ & $8 \times 10^{-4}$ & $6 \times 10^{-5}$ & $9 \times 10^{-6}$ & $4 \times 10^{-6}$ & \\
\hline & & $\Delta E^{[3]}$ & $2 \times 10^{-3}$ & $7 \times 10^{-4}$ & $6 \times 10^{-5}$ & $9 \times 10^{-6}$ & $4 \times 10^{-6}$ & \\
\hline $\mathrm{H}_{2}\left({ }^{1} S\right)$ & $-1.1336286746^{\mathrm{c}}$ & $\Delta E^{[0]}$ & $1 \times 10^{-2}$ & $3 \times 10^{-3}$ & $3 \times 10^{-4}$ & $1 \times 10^{-5}$ & $2 \times 10^{-6}$ & $+2 \times 10^{-6}$ \\
\hline & & $\Delta E^{[1]}$ & $2 \times 10^{-4}$ & $4 \times 10^{-5}$ & $4 \times 10^{-6}$ & $4 \times 10^{-8}$ & $8 \times 10^{-9}$ & \\
\hline & & $\Delta E^{[2]}$ & $9 \times 10^{-5}$ & $2 \times 10^{-6}$ & $3 \times 10^{-8}$ & & & \\
\hline & & $\Delta E^{[3]}$ & $9 \times 10^{-5}$ & $2 \times 10^{-6}$ & $3 \times 10^{-8}$ & & & \\
\hline $\mathrm{Li}\left({ }^{2} S\right)$ & -7.43275092 & $\Delta E^{[0]}$ & $2 \times 10^{-2}$ & $5 \times 10^{-3}$ & $2 \times 10^{-3}$ & $7 \times 10^{-4}$ & $8 \times 10^{-5}$ & $+3 \times 10^{-5}$ \\
\hline & & $\Delta E^{[1]}$ & $3 \times 10^{-4}$ & $4 \times 10^{-5}$ & $5 \times 10^{-5}$ & $4 \times 10^{-6}$ & $3 \times 10^{-7}$ & \\
\hline & & $\Delta E^{[2]}$ & $5 \times 10^{-6}$ & $3 \times 10^{-7}$ & $2 \times 10^{-6}$ & $2 \times 10^{-7}$ & $3 \times 10^{-8}$ & \\
\hline & & $\Delta E^{[3]}$ & $5 \times 10^{-6}$ & $4 \times 10^{-7}$ & $2 \times 10^{-6}$ & $2 \times 10^{-7}$ & $3 \times 10^{-8}$ & \\
\hline $\operatorname{Be}\left({ }^{1} S\right)$ & $-14.57302317^{\mathrm{d}}$ & $\Delta E^{[0]}$ & $4 \times 10^{-2}$ & $8 \times 10^{-3}$ & $2 \times 10^{-3}$ & $6 \times 10^{-4}$ & $1 \times 10^{-4}$ & $+9 \times 10^{-5}$ \\
\hline & & $\Delta E^{[1]}$ & $1 \times 10^{-3}$ & $3 \times 10^{-5}$ & $3 \times 10^{-5}$ & $2 \times 10^{-6}$ & $3 \times 10^{-7}$ & \\
\hline & & $\Delta E^{[2]}$ & $4 \times 10^{-5}$ & $3 \times 10^{-6}$ & $4 \times 10^{-6}$ & $2 \times 10^{-7}$ & $2 \times 10^{-8}$ & \\
\hline & & $\Delta E^{[3]}$ & $4 \times 10^{-5}$ & $3 \times 10^{-6}$ & $3 \times 10^{-6}$ & $1 \times 10^{-7}$ & $2 \times 10^{-8}$ & \\
\hline $\mathrm{N}\left({ }^{4} S\right)$ & -54.404548301 & $\Delta E^{[0]}$ & $2 \times 10^{-1}$ & $3 \times 10^{-2}$ & $4 \times 10^{-3}$ & $5 \times 10^{-4}$ & $4 \times 10^{-5}$ & $-8 \times 10^{-6}$ \\
\hline & & $\Delta E^{[1]}$ & $3 \times 10^{-3}$ & $3 \times 10^{-4}$ & $2 \times 10^{-5}$ & $7 \times 10^{-7}$ & $3 \times 10^{-8}$ & \\
\hline & & $\Delta E^{[2]}$ & $2 \times 10^{-4}$ & $3 \times 10^{-5}$ & $3 \times 10^{-6}$ & $7 \times 10^{-8}$ & $2 \times 10^{-9}$ & \\
\hline & & $\Delta E^{[3]}$ & $2 \times 10^{-4}$ & $3 \times 10^{-5}$ & $3 \times 10^{-6}$ & $6 \times 10^{-8}$ & $2 \times 10^{-9}$ & \\
\hline $\mathrm{Ne}\left({ }^{1} S\right)$ & -128.547098108 & $\Delta E^{[0]}$ & $5 \times 10^{-1}$ & $1 \times 10^{-1}$ & $9 \times 10^{-3}$ & $7 \times 10^{-4}$ & $5 \times 10^{-5}$ & $-7 \times 10^{-7}$ \\
\hline & & $\Delta E^{[1]}$ & $4 \times 10^{-3}$ & $4 \times 10^{-4}$ & $2 \times 10^{-5}$ & $4 \times 10^{-7}$ & $5 \times 10^{-8}$ & \\
\hline & & $\Delta E^{[2]}$ & $5 \times 10^{-4}$ & $6 \times 10^{-5}$ & $2 \times 10^{-6}$ & $1 \times 10^{-7}$ & $2 \times 10^{-9}$ & \\
\hline & & $\Delta E^{[3]}$ & $5 \times 10^{-4}$ & $7 \times 10^{-5}$ & $2 \times 10^{-6}$ & $1 \times 10^{-7}$ & $2 \times 10^{-9}$ & \\
\hline
\end{tabular}

${ }^{\mathrm{a}}$ Reference 37.

${ }^{\mathrm{b}}$ Reference 33 .

${ }^{c}$ Reference 38.

${ }^{\mathrm{d}}$ Reference 39 .

markably accurate HFPC energies. The best of these, HFPC [3]/pc- 0 , is only $28 \mu E_{h}$ above the HF limit and is, in fact, competitive with the $\mathrm{HF} / \mathrm{pc}-3$ energy. We found that $\mathrm{HFPC}[3] / \mathrm{pc}-1$ is comparable to $\mathrm{HF} / \mathrm{pc}-4$ and that HFPC[3]/ pc-3 misses the CBS limit by only 20 picohartree.

\section{Triplet He atom}

The Rydberg orbitals of helium are very diffuse ${ }^{33}$ and it is not surprising to find that the HF/pc- $n$ energies of the $1 s 2 s$ triplet state are poor. Yet, Table II shows that HFPC is capable of yielding accurate energies, even from these bad starting points. The HF/pc-0 energy is too high by roughly 1 hartree, but the HFPC $[1] / \mathrm{pc}-0$ error is only $80 \mathrm{~m} E_{h}$, which is comparable to $\mathrm{HF} / \mathrm{pc}-3$. The self-interaction error is evidently significant because, when we move to HFPC $[2] / \mathrm{pc}-0$, the error drops to $2 \mathrm{~m} E_{h}$. The pattern is similar for larger primary basis sets and suggests that HFPC is an effective tool for improving both crude and accurate HF calculations. It is reassuring to find that the HFPC [2] and HFPC [3] errors are invariably close, for this indicates that the nonorthogonality errors in HFPC[2] are small.

\section{D. $\mathrm{H}_{2}$ molecule}

We used the bond length $R=1.4 \mathrm{bohr}$, targeting the HF energy at the $s p d$-limit using Cartesian $d$ functions and dropping the $f$ and $g$ functions from the pc-3 and pc-4 bases. Table II shows that HFPC[3] gives improvements of two, three, and four orders of magnitude when used with the pc-0, pc-1, and pc-2 basis sets, respectively, and the HFPC[3]/pc-2 error is a mere $28 n E_{h}$. We are particularly encouraged by the accuracy of HFPC[3]/pc-0 because this basis contains only two $s$ functions per hydrogen atom. To the extent that we can generalize from this result, it suggests that accurate HFPC results for molecular systems may not require a polarized primary basis. This contrasts with the dual-basis (DBHF) findings of Steele et al. ${ }^{17}$ but these authors systematically ignore terms that are quadratic in the density matrix improvement.

To explore this, we used DBHF and HFPC [1] to estimate the $\mathrm{HF} / 6-311++\mathrm{G}(3 d f, 3 p d)$ energy of the $\mathrm{H}_{2}$ molecule. Using $6-311 \mathrm{G}^{*}$ as the primary basis, ${ }^{17}$ DBHF gives an error of $0.7 \mathrm{~m} E_{h}$. Remarkably, using STO-3G as the primary basis, HFPC $[1]$ gives an error of $0.3 \mathrm{~m} E_{h}$. 


\section{E. Larger atoms}

The application of HFPC to $\mathrm{Li}, \mathrm{Be}, \mathrm{N}$, and $\mathrm{Ne}$ atoms yields HFPC [1] energies that are typically two orders of magnitude more accurate than the corresponding HF energies and HFPC[2] energies that are usually one or two orders of magnitude more accurate again. As we also observed for the triplet $\mathrm{He}$ atom, the $\mathrm{HFPC}[3]$ results indicate that the nonorthonormality of the HFPC[2] orbitals has negligible energetic effects.

\section{CONCLUDING REMARKS}

HFPCs are noniterative approaches that improve a finitebasis HF calculation on a given molecule toward the CBS limit. We have shown that they yield significant improvements even if the primary basis is only of moderate quality and our results suggest that, in the best cases, they offer a quadratic reduction in the relative error. We will present a theoretical rationalization of this unexpectedly good result in a forthcoming paper.

We found that HFPC $[1] / \mathrm{pc}-0$ is usually more accurate than conventional $\mathrm{HF} / \mathrm{pc}-2$ and it follows, for example, that HFPC [1] may allow HF energies of triple-zeta quality to be obtained cheaply from single-zeta calculations, a possibility with far-reaching practical applications.

The more complex corrections, HFPC[2] and HFPC[3], are even more accurate but require the formation of multiple Fock matrices and are necessarily more expensive. We are implementing all three methods in the Q-CHEM package and will report comprehensive accuracy and timing benchmarking on larger systems elsewhere. HFPC is a rigorous alternative to basis set extrapolation and should be useful in future high-level composite methods such as the HEAT, ${ }^{34} \mathrm{Wn},{ }^{35}$ and $\mathrm{G} n$ (Ref. 36) families.

\section{ACKNOWLEDGMENTS}

We thank Taweetham Limpanuparb for programming assistance and Professor Frank Jensen for the kind supply of basis sets. J.D. thanks the ANU for a Ph.D. scholarship and P.M.W.G. thanks the Australian Research Council for funding (Grant No. DP0771978).

\footnotetext{
${ }^{1}$ E. Schrödinger, Ann. Phys. 384, 361 (1926).

${ }^{2}$ N. L. Allinger, J. T. Fermann, W. D. Allen, and H. F. Schaefer III, J. Chem. Phys. 106, 5143 (1997).

${ }^{3}$ T. Helgaker, W. Klopper, H. Koch, and J. Noga, J. Chem. Phys. 106, 9639 (1997)

${ }^{4}$ J. M. L. Martin, J. Mol. Struct.: THEOCHEM 398-399, 135 (1997).
}

${ }^{5}$ A. Halkier, P. Jorgensen, W. Klopper, and J. Olsen, Chem. Phys. Lett. 302, 437 (1999).

${ }^{6}$ S. B. Huh and J. S. Lee, J. Chem. Phys. 118, 3035 (2003).

${ }^{7}$ F. Jensen, Theor. Chem. Acc. 113, 267 (2005).

${ }^{8}$ A. J. C. Varandas, J. Chem. Phys. 126, 244105 (2007).

${ }^{9}$ D. Bakowies, J. Chem. Phys. 127, 164109 (2007).

${ }^{10}$ S. Zhong, E. C. Barnes, and G. A. Petersson, J. Chem. Phys. 129, 184116 (2008).

${ }^{11}$ S. Havriliak and H. F. King, J. Am. Chem. Soc. 105, 4 (1983).

${ }^{12}$ S. Havriliak, T. R. Furlani, and H. F. King, Can. J. Phys. 62, 1336 (1984).

${ }^{13}$ R. Jurgens-Lutovsky and J. Almlöf, Chem. Phys. Lett. 178, 451 (1991).

${ }^{14}$ K. Wolinski and P. Pulay, J. Chem. Phys. 118, 9497 (2003).

${ }^{15}$ W. Liang and M. Head-Gordon, J. Phys. Chem. A 108, 3206 (2004).

${ }^{16}$ R. P. Steele, R. A. DiStasio, Jr., Y. Shao, J. Kong, and M. Head-Gordon, J. Chem. Phys. 125, 074108 (2006).

${ }^{17}$ R. P. Steele, Y. Shao, R. A. DiStasio, Jr., and M. Head-Gordon, J. Phys. Chem. A 110, 13915 (2006).

${ }^{18}$ R. P. Steele and M. Head-Gordon, Mol. Phys. 105, 2455 (2007).

${ }^{19}$ A. Szabo and N. S. Ostlund, Modern Quantum Chemistry (McGraw-Hill, New York, 1989).

${ }^{20}$ C. C. J. Roothaan, Rev. Mod. Phys. 23, 69 (1951).

${ }^{21}$ G. G. Hall, Proc. R. Soc. London A205, 541 (1951).

${ }^{22}$ J. A. Pople and R. K. Nesbet, J. Chem. Phys. 22, 571 (1954).

${ }^{23}$ P. M. W. Gill, Adv. Quantum Chem. 25, 141 (1994).

${ }^{24}$ J. P. Perdew and A. Zunger, Phys. Rev. B 23, 5048 (1981).

${ }^{25}$ F. Jensen, J. Chem. Phys. 115, 9113 (2001).

${ }^{26}$ F. Jensen, J. Chem. Phys. 116, 7372 (2002).

${ }^{27}$ F. Jensen, J. Phys. Chem. A 111, 11198 (2007).

${ }^{28}$ Y. Shao, L. Fusti-Molnar, Y. Jung, J. Kussmann, C. Ochsenfeld, S. T. Brown, A. T. B. Gilbert, L. V. Slipchenko, S. V. Levchenko, D. P. O’Neill, R. A. DiStasio, Jr., R. C. Lochan, T. Wang, G. J. O. Beran. N. A. Besley, J. M. Herbert, C Y Lin, T. Van Voorhis, S. H. Chien, A. Sodt, R. P. Steele, V. A. Rassolov, P. E. Maslen, P. P. Korambath, R. D. Adamson, B. Austin, J. Baker, E. F. C. Byrd, H. Dachsel, R. J. Doerksen, A. Dreuw, B. D. Dunietz, A. D. Dutoi, T. R. Furlani, S. R. Gwaltney, A. Heyden, S. Hirata, C. P. Hsu, G. Kedziora, R. Z. Khalliulin, P. Klunzinger, A. M. Lee, M. S. Lee, W. Z. Liang, I. Lotan, N. Nair, B. Peters, E. I. Proynov, P. A. Pieniazek, Y. M. Rhee, J. Ritchie, E. Rosta, C. D. Sherrill, A. C. Simmonett, J. E. Subotnick, H. L. Woodcock III, W. Zhang, A. T. Bell, A. K. Chakraborty, D. M. Chipman, F. J. Keil, A. Warshel, W. J. Hehre, H. F. Schaefer III, J. Kong, A. I. Krylov, P. M. W. Gill, and M. HeadGordon, Phys. Chem. Chem. Phys. 8, 3172 (2006).

${ }^{29}$ MATHEMATICA, Version 6.0, Wolfram Research, Inc., 2007.

${ }^{30}$ M. W. Schmidt and K. Ruedenberg, J. Chem. Phys. 71, 3951 (1979).

${ }^{31} \mathrm{~F}$. Jensen, private communication (October 1, 2008).

${ }^{32}$ B. Klahn and W. A. Bingel, Theor. Chim. Acta 44, 27 (1977).

${ }^{33}$ J. Deng, A. T. B. Gilbert, and P. M. W. Gill, Int. J. Quantum Chem. 109, 1915 (2009).

${ }^{34}$ A. Tajti, P. G. Szalay, A. G. Csaszar, M. Kallay, J. Gauss, and E. F. Valeev, J. Chem. Phys. 121, 11599 (2004).

${ }^{35}$ A. Karton, E. Rabinovich, and J. M. L. Martin, J. Chem. Phys. 125, 144108 (2006).

${ }^{36}$ L. A. Curtiss, P. C. Redfern, and K. Raghavachari, J. Chem. Phys. 126, 084108 (2007).

${ }^{37}$ K. Szalewicz and H. J. Monkhorst, J. Chem. Phys. 75, 5785 (1981).

${ }^{38}$ F. Jensen, J. Chem. Phys. 110, 6601 (1999).

${ }^{39}$ T. Koga, Theor. Chim. Acta 95, 113 (1997). 